\title{
Clinical Pearls and Advances in Molecular Researches of Epilepsy-Associated Tumors
}

\author{
Ji Hoon Phi, M.D., Ph.D., Seung-Ki Kim, M.D., Ph.D. \\ Division of Pediatric Neurosurgery, Seoul National University Children's Hospital, Seoul, Korea
}

Brain tumors are the second most common type of structural brain lesion that causes chronic epilepsy. Patients with low-grade brain tumors often experience chronic drug-resistant epilepsy starting in childhood, which led to the concept of long-term epilepsy-associated tumors (LEATs). Dysembryoplastic neuroepithelial tumor and ganglioglioma are representative LEATs and are characterized by young age of onset, frequent temporal lobe location, benign tumor biology, and chronic epilepsy. Although highly relevant in clinical epileptology, the concept of LEATs has been criticized in the neuro-oncology field. Recent genomic and molecular studies have challenged traditional views on LEATs and low-grade gliomas. Molecular studies have revealed that lowgrade gliomas can largely be divided into three groups : LEATs, pediatric-type diffuse low-grade glioma (DLGG; astrocytoma and oligodendroglioma), and adult-type DLGG. There is substantial overlap between conventional LEATs and pediatric-type DLGG in regard to clinical features, histology, and molecular characteristics. LEATs and pediatric-type DLGG are characterized by mutations in BRAF, FGFR1, and MYB/MYBL1, which converge on the RAS-RAF-MAPK pathway. Gene (mutation)-centered classification of epilepsyassociated tumors could provide new insight into these heterogeneous and diverse neoplasms and may lead to novel molecular targeted therapies for epilepsy in the near future.

Key Words : Brain neoplasms · Seizures · Epilepsy · Glioma · Classification.

\section{INTRODUCTION}

Epilepsy is a chronic neurological disorder affecting many children worldwide. A significant portion of patients have drug-resistant epilepsy that requires a careful work-up and surgical considerations. Focal structural abnormalities in the brain can be defined by high-resolution magnetic resonance imaging (MRI) and other imaging modalities. Extirpation of the lesion provides a high probability of long-term seizure control. Brain tumors are one of the most common structural lesions associated with seizures and epilepsy. As early as 1885 , William Osler reported to the Boston Medical and Surgical Journal about a teenage girl who suffered from Jacksonian seizures for 14 years and finally died of status epilepticus ${ }^{21}$. Through an autopsy of her brain, Osler found an almondsized 'glioma' under her right motor cortex. Although we do not know the exact diagnosis of the tumor (i.e., the type of glioma) in current nosologic terms, the early age of seizure onset, protracted clinical course, and small nodular features of the lesion suggest that the tumor was a kind of low-grade glioma,

- Received : January 25, 2019 •Revised : March 7, 2019 •Accepted : March 17, 2019

- Address for reprints : Seung-Ki Kim, M.D., Ph.D.

Division of Pediatric Neurosurgery, Seoul National University Children's Hospital, 101 Daehak-ro, Jongno-gu, Seoul 03080, Korea

Tel : +82-2-2072-3084, Fax : +82-2-744-8459, E-mail : nsthomas@snu.ac.kr

This is an Open Access article distributed under the terms of the Creative Commons Attribution Non-Commercial License (http://creativecommons.org/licenses/by-nc/4.0) which permits unrestricted non-commercial use, distribution, and reproduction in any medium, provided the original work is properly cited. 
such as ganglioglioma. This case confirmed that chronic epilepsy can be caused by a brain tumor near the eloquent cortex and revealed the slow-growing, indolent nature of epilepsy-associated 'glioma'.

Our knowledge of brain tumors and epilepsy has greatly evolved since Osler's time. With the advent of neuroimaging techniques, the diagnosis and treatment of brain tumors and epilepsy have been revolutionized. Furthermore, recent advances in molecular genomics urge us to question the basic terms and concepts of neuropathology. For example, what is an astrocyte, and what is astrocytoma? Research has also revealed the hidden nature of epilepsy-associated tumors and their epileptogenic mechanism. This article aims to provide an overview of the evolving landscape of epilepsy-associated tumors, focusing on the pathological and molecular characteristics. The development of and challenges related to the treatment of epilepsy-associated tumors are also considered.

\section{LONG-TERM EPILEPSY-ASSOCIATED BRAIN TUMORS (LEATS)}

Brain tumors are the second most common structural lesion associated with epilepsy. Data from the European Epilepsy Brain Bank (EEBB), which contains 9532 epilepsy surgery specimens showed that brain tumors constituted $23.6 \%$ of the causative diagnoses, next to only hippocampal sclerosis $(36.4 \%)^{6}$. Virtually all brain tumors can elicit seizure attacks that, if recurrent, lead to epilepsy. Actually, seizure is one of the major symptoms of meningiomas ${ }^{3}$, and even cerebellar tumors can be the source of seizures ${ }^{23}$. However, intra-axial brain tumors involving the cerebral cortex are more closely related to epilepsy than extra-axial tumors or deep-seated intra-axial tumors. Many studies have consistently shown that low-grade brain tumors are more likely to cause epileptic seizures than high-grade brain tumors such as glioblastoma ${ }^{37}$. High-grade tumors tend to damage surrounding structures (including potential epileptogenic areas), whereas low-grade tumors may displace or distort nearby neurons and neuronal networks, evoking seizures.

Some low-grade brain tumors are more frequently involved in chronic intractable epilepsy. Hence, the concept of so-called LEATs was introduced by the Bonn Epilepsy Center ${ }^{31)}$. Ganglioglioma and dysembryoplastic neuroepithelial tumor
(DNET) are representative tumors of LEATs. Ganglioglioma alone accounted for $10.4 \%$, and DNET constituted $5.9 \%$ of the EEBB cohor ${ }^{6}$. Minor subtypes of LEATs include pleomorphic xanthoastrocytoma (PXA), angiocentric glioma, and isomorphic astrocytoma. LEATs, typically ganglioglioma and DNET, are characterized by early age of diagnosis (children and adolescents), the predilection to temporal lobe, cortical involvement, and low probability of malignant progression. Most LEATs are so-called glioneuronal tumors that exhibit both glial and neuronal differentiation patterns. The World Health Organization (WHO) classification of brain tumors also places individual LEATs in mixed neuronal and glial tumor group (but the WHO never officially accepted the term LEATs) ${ }^{11}$. One notable characteristic of LEATs is their close relationship with brain cortical dysgenesis. An original description of DNET was a composite tumor consisting of a tumor with specific glioneuronal elements and an adjacent focal cortical dysplasia (FCD) (so-called 'complex type') ${ }^{16}$. Later, a simple type with only glioneuronal elements and nonspecific variants (although highly controversial) without glioneuronal elements was added to the DNET category ${ }^{15,17)}$. The coexistence of FCD is also commonly observed in ganglioglioma ${ }^{42}$. It is also intriguing that some patients develop a composite neoplasm consisting of DNET and ganglioglioma together ${ }^{433}$. Therefore, the current classification of brain cortical malformation regards DNET and ganglioglioma as a kind of brain malformation (malformations secondary to abnormal neuronal and glial proliferation), alongside FCD and hemimegalencephaly ${ }^{2}$. The frequent temporal lobe involvement, cortical location, low-grade pathology, and association with FCD all appear to contribute to the high propensity of LEATs to cause chronic drug-resistant epilepsy.

Although relevant in clinical practice, several aspects of the concept of LEATs have been questioned. First, the term was originally applied to brain tumors associated with long-term (usually $>2$ years), drug-resistant epilepsy ${ }^{31}$. However, since the time that the term was proposed, the definition of medical intractability in epilepsy has become less stringent. In particular, in pediatric epilepsy, the strategy of an early imaging work-up and surgery, if possible, has been encouraged to prevent the neurocognitive deficits caused by recurrent seizures and the resulting prevention of normal development ${ }^{24}$. In this regard, changing the phrase 'long-term' in LEATs to 'lowgrade' has been proposed, as the majority of LEATs are truly 
low-grade neoplasms ${ }^{4}$.

Second, LEATs are not an established concept. PXAs, once included in LEATs, are now considered to be diffuse gliomas (as opposed to LEATs), mainly because PXA has far more aggressive biological behavior than typical LEATs. From a prognostic perspective, one fourth of PXAs are of a high grade (WHO grade III or IV) and the 10-year overall survival of PXA patients was only $67 \%{ }^{39)}$. Angiocentric glioma is a newly adopted member of the WHO classification of brain tumors. Angiocentric glioma develops in the brain cortex of children, resembles ependymoma histologically, and has proven to be highly epileptogenic, designating the tumor as a legitimate LEAT $^{35)}$. However, angiocentric glioma is a rare tumor with an unknown overall incidence. So-called isomorphic astrocytoma is an intriguing entity with distinct histopathology and excellent prognosis compared with ordinary diffuse astrocytoma, but its validity as a separate entity has not been accepted by neuro-oncology societies ${ }^{5)}$.

Furthermore, significant pathological and clinical heterogeneity exists in each tumor category. DNETs are highly likely to be associated with chronic epilepsy, but many patients with DNET exhibit only sporadic seizures, no seizures, or even no symptoms. The majority of gangliogliomas are benign, lowgrade tumors, but some tumors exhibit anaplasia in histology and become frankly malignant over the clinical course. Therefore, although widely accepted by epilepsy societies, LEATs have never been formally included in the WHO classification of brain tumor pathology. This discrepancy between clinical features (chronic epilepsy) and pathological coherency (diverse entities) may be compromised by novel findings in genomic studies on LEATs, which will be discussed in a later section.

\section{DIFFUSE LOW-GRADE GLIOMA (DLGG) AND EPILEPSY}

DLGG includes astrocytoma and oligodendroglioma. These tumors commonly develop in young adults. DLGG involves large areas of brain cortex and subcortical areas, most notably the frontal lobes. Seizure and epilepsy are the most common manifestations of DLGG. Nearly $80-90 \%$ of patients with DLGG develop seizures ${ }^{36,37)}$. The so-called 'low-grade' glioma incorporates both DLGG and LEATs. The majority of DLGGs correspond to histopathological grade II tumors with a far higher rate of infiltration, recurrence, and malignant transformation than typical LEATs, which are mostly grade I tumors. Frequently, DLGG has been excluded from the discussion of epilepsy-associated tumors (the EEBB cohort mentioned above does not include DLGG cases) because DLGG is considered a bona fide invasive glioma that should be dealt with in the oncology field ${ }^{6}$. However, many patients with DLGG attain long-term survival and subsequently face the same problem of long-standing epilepsy as patients with LEATs. In fact, any epilepsy surgery cohort must include a number of patients with DLGG in addition to the backbone of the LEAT group, especially when adolescents and young adults are included ${ }^{40)}$.

To make the situation more complex, DLGG can be roughly divided into so-called adult-type tumors and pediatric-type tumors, according to the mutation profiles. For example, pediatric-type astrocytomas lack IDH1/2 mutations and rarely undergo malignant transformation. So-called pediatric-type oligodendrogliomas are frequently devoid of IDH1/2 mutation and $1 \mathrm{p} / 19 \mathrm{q}$ codeletion, the hallmarks of oligodendroglio$\mathrm{ma}^{29,45}$. A study re-evaluated 100 cases of histologically-diagnosed oligodendrogliomas from patients under the age of 20 years $^{48}$. In the study, a pathology review revealed that only 50 cases were considered oligodendrogliomas and the others were reclassified as DNET, gangliocytoma, ependymoma, and astrocytoma. In the 50 cases of oligodendrogliomas, IDH1 mutation and $1 \mathrm{p} / 19 \mathrm{q}$ codeletion were found in only $18 \%$ and $25 \%$ of cases, respectively ${ }^{48}$. It is difficult to diagnose tumors with oligodendrocyte-like cells because many low-grade gliomas share histologic features. There is certainly a fair amount of overlap between the pediatric-type DLGGs and LEATs on pathological and clinical grounds. Therefore, to evaluate the true pathological spectrum of epilepsy-associated tumors, molecular classification should be considered.

Clinically, oligodendrogliomas in children tends to be smaller and more commonly found in the temporal lobe than their adult counterparts which develop most frequently in the frontal lobe ${ }^{222}$. Pediatric oligodendrogliomas has far better prognosis than adult tumors of the same histology ${ }^{22}$. The different molecular characteristics of pediatric-type and adult-type DLGG may underlie this phenomenon. Erroneous classification and inclusion of LEATs (esp., DNET and ganglioglioma), as discussed above, may also contribute the better survival of pediatric patients. 


\section{MOLECULAR LANDSCAPE OF EPILEPSY-ASSO- CIATED TUMORS}

Recent rapid progress in sequencing technology and the refined classification based on genomic signatures have allowed unprecedented opportunities to understand brain tumors, especially low-grade gliomas. In pediatric low-grade gliomas, whole genome sequencing revealed that most pediatric lowgrade gliomas have alterations of BRAF, FGFR, and MYB/ MYBL1 genes ${ }^{54)}$. In particular, pediatric DLGG showed either duplication of the tyrosine kinase domain of the FGFR1 gene or rearrangement of the MYB/MYBL1 genes. Mutations of IDH1/2, the hallmark of adult-type DLGG were rarely found in pediatric DLGG, supporting the distinction of pediatrictype DLGG from its adult counterpart ${ }^{46,54)}$. Mutations of TP53 and ATRX, the other common variations in adult-type diffuse astrocytomas were also rarely found in pediatric-type DLGG.

In LEAT group, alterations of the BRAF gene, notably the BRAF V600E mutation is highly represented in gangliogliomas $^{18)}$. In 36 gangliogliomas that were sequenced, 27 tumors (75\%) had a BRAF V600E mutation, variant BRAF mutation, or BRAF fusion. The other tumors had mutations in KRAS, RAF, NF1, or an FGFR1/2 alteration, confirming that ganglioglioma develops through alterations in the RAS-RAF-MAPK pathway $^{38)}$. A study showed that mutated BRAF V600E protein was predominantly expressed in neuronal lineage cells, although BRAF was expressed in both the neuronal and glial compartments ${ }^{27}$. Interestingly, when the BRAF V600E pathogenic mutation was introduced into mouse embryos, the embryos postnatally developed an increased neuronal size and epileptic phenotype, whereas the same mutation transfected into postnatal mouse brains led to glial proliferation only, indicating that BRAF V600E induced epileptogenesis in the neuronal lineage and tumorigenesis in the glial lineage ${ }^{28}$.

A large-scale study on DNET revealed that alterations of FGFR1 (duplication or point mutation) were predominant (58\%) with no observed cases of BRAF alterations ${ }^{47}$. Previous small-scale studies reported the presence of BRAF alterations in up to $30 \%$ of $\mathrm{DNET}^{8,34)}$. The discrepancy in BRAF alterations can be explained by the fact that examining 'nonDNET' (DNET tissues that do not meet the strict WHO criteria in a central pathology review) revealed a lower frequency (19\%) of FGFR1 mutations and a higher proportion (13\%) of BRAF V600E mutations ${ }^{47}$. The interobserver reliability of his- topathological diagnoses of LEATs has been questioned ${ }^{50)}$. Many glioneuronal tumors lack specific histological features that are crucial for the diagnosis of ganglioglioma or DNET, making diagnosis difficult. Immunohistochemistry with staining for CD34, S100, and synaptophysin is helpful, but the markers are essentially nonspecific ${ }^{51}$. Some researchers have tried to extend the definition of DNET to include these nonspecific, aberrant glioneuronal tumors ${ }^{17)}$. Recently, Stone et al. ${ }^{50)}$ found that LEATs were roughly divided into two molecular groups, regardless of their histology. After a histologic review of archived LEAT tissues, they reclassified them into three groups : ganglioglioma, DNET, and glioneuronal tumor not otherwise specified. Expression and methylation profiling segregated all cases into two molecular groups, one predominantly with ganglioglioma (group 1) and the other mainly with DNET (group 2). It is noteworthy that each group included both ganglioglioma and DNET in different proportions. As expected, group 1 was enriched for the BRAF V600E mutation, and group 2 was enriched for FGFR1 alterations. Qaddoumi et al. ${ }^{44)}$ also reported similar findings: diverse lowgrade glioneuronal tumors were segregated into BRAF-group/ ganglioglioma-like tumors, FGFR1 group/tumors with oligodendrocyte-like cells (including DNET), and MYB group/astrocytoma-like tumors. A small but non-negligible overlap existed between the molecular groups. These studies revealed that the histological differentiation of LEATs (and DLGG) is incomplete and that molecular profiling can reveal the fundamental characteristics of the tumors, enabling more precise diagnosis and classification.

\section{TREATMENT OF TUMOR-RELATED EPILEPSY IN THE MOLECULAR ERA}

In tumor-related epilepsy, treatment should have two aims : tumor control and seizure freedom ${ }^{40)}$. Because the majority of LEATs are grade I tumors, complete surgical resection confers excellent long-term tumor control. According to the Surveillance, Epidemiology, and End Results (SEER) database of pediatric patients with ganglioglioma/gangliocytoma, who were treated between 2004 and 2010, two-thirds of patients received gross total resection (GTR). The five-year overall survival of patients with non-brainstem tumors was $96.6 \%{ }^{19)}$. Despite excellent overall survival, progression-free survival was far lower 
( $37 \%$ at 10 years in one study), indicating that recurrence is not uncommon $^{13}$. It should be noted that ganglioglioma has some malignant potential, usually after recurrence, although it is rare in children ${ }^{26,33}$. GTR is the most important factor for long-term tumor control of ganglioglioma, as in other low-grade tumors. Previously, it was suggested that DNET has a low likelihood of recurrence even after subtotal resection ${ }^{16)}$. However, recent studies have indicated that DNET has a higher recurrence potential than previously thought ${ }^{14,32}$. Some DNETs have poor delineation and gray-white matter blurring around the tumor on MRI, indicating the existence of adjacent $\mathrm{FCD}^{10)}$. GTR of this type of DNET has proven difficult ${ }^{9}$. In a meta-analysis of DNET treatment, the median GTR rate was 79\% (interquartile range $62-92 \%)^{7}$. Many studies did not report the actual recurrence rate as documented on MRI but only mentioned the reoperation rate, which ranged from $5-46 \%{ }^{7}$. DNET tends to grow very slowly even after recurrence, frequently without symptoms. Reoperation is also inadequate to evaluate the true oncological properties of DNETs, because reoperation may be necessary to control seizures regardless of tumor recurrence. In a study evaluating the radiological recurrence of LEATs, the long-term recurrence-free survival of DNET was approximately $75 \%{ }^{52)}$

Seizure freedom after surgery was variable, but the data suggested that there was $80-85 \%$ long-term ( $>5$ years) seizure control $^{20,31,40)}$. GTR of the lesion was also an important factor affecting seizure control, but other clinical factors, such as shorter duration of epilepsy or the absence of secondary generalization, were associated with better outcomes in some studies ${ }^{20}$. Although factors affecting seizure control are more complex than tumor control, it seems obvious that the two outcomes are very closely related. Seizure recurrence is commonly the first sign of tumor recurrence. Pallud and McKhann ${ }^{37)}$ provided relevant examples in which seizure frequency was attenuated according to a decrease in tumor burden. Seizure recurrence was significantly more frequent for DNET than for ganglioglioma ${ }^{52}$. Seizure control seems less favorable for DLGG than for LEATs. Long-term seizure control for diffuse astrocytoma was $66 \%$ in the Bonn series ${ }^{31}$. In the Beijing series, one-year seizure freedom was $65 \%$ after resection of $D_{L G G}{ }^{33}$. The former study included both pediatric and adult patients, and the latter series is composed of only adult patients. The median duration of epilepsy was far longer in the Bonn series than in the Beijing series (12 years vs. 10 months), reflecting the different tumor biology of the subjects of each study. The unfavorable seizure outcome of DLGG may be attributed to the diffuse, invasive nature of DLGG and the higher probability of incomplete resection ${ }^{311}$.

There has long been an unsettled controversy over whether

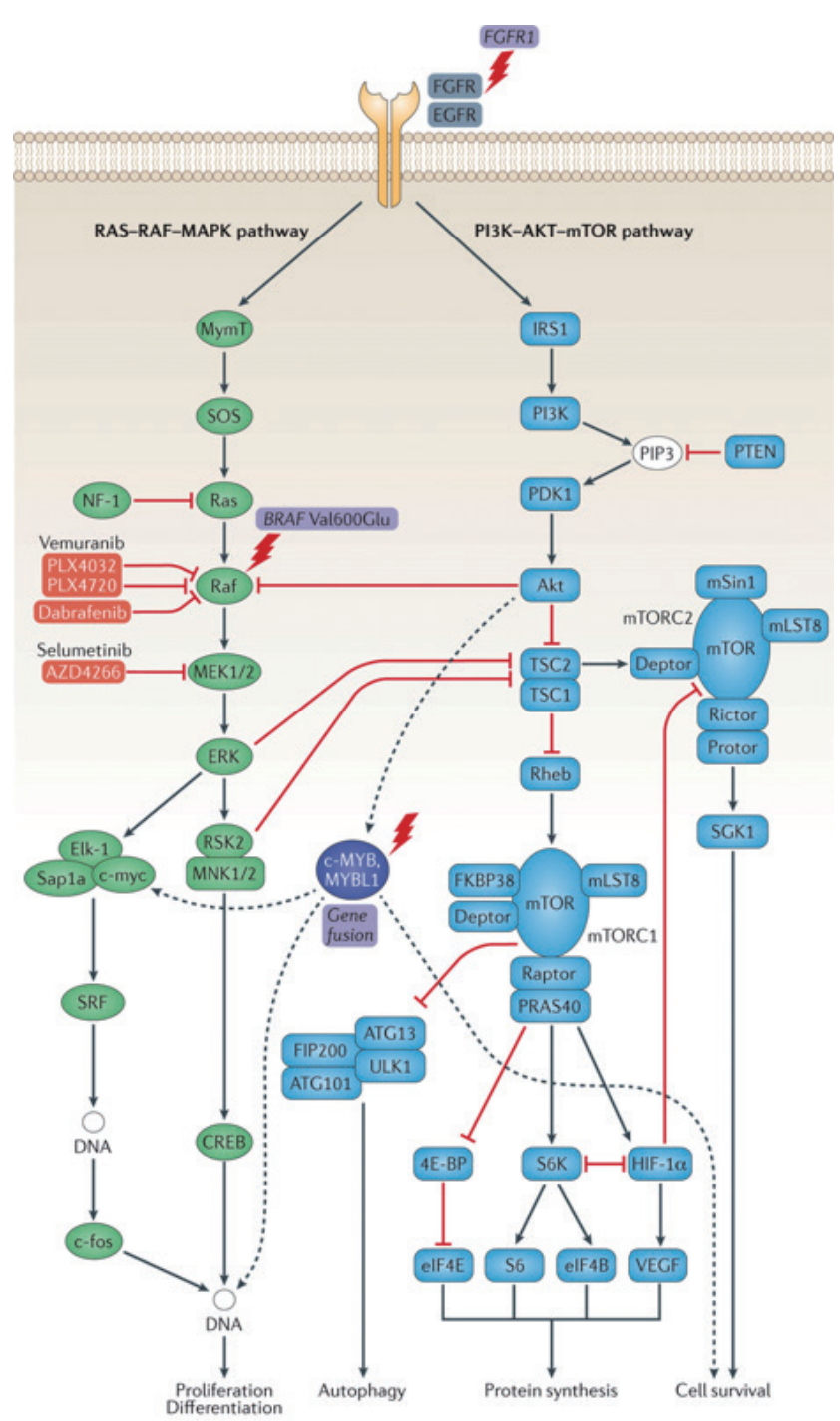

Fig. 1. Molecular genetic pathways in long-term epilepsy-associated tumors (LEATs) and pediatric type diffuse low-grade glioma (DLGG). Two major signaling pathways, the RAS-RAF-MAPK (green) and PI3K-AKT-mTOR (blue) play major role in LEATs. Upstream in receptor signaling, FGFR1 mutations have been described in dysembryoplastic neuroepithelial tumors. Activation of RAS-RAF-MAPK pathway by BRAF V600E mutation is frequently detected in gangliogliomas. BRAF and MEK1/2 are pharmacological targets for therapy (pink). MAPK activation is regulated by substrates of the PI3KAKT-mTOR signaling cascade (red links). c-MYB/MYBL1 (purple) is altered in angiocentric gliomas. Genetic alterations described in LEATs are designated by a lightning bolt. Reprint from Blümcke et al. ${ }^{4}$ with permission from Springer Nature. 
tumor resection alone (lesionectomy) or extended lesionectomy (epilepsy surgery including nearby epileptogenic cortex) yields superior seizure control. The surgical strategy differs greatly from center to center, and there is no clear evidence supporting either strategy, partly because the patients and pathologic spectrum of tumor-related epilepsy are too heterogeneous ${ }^{40)}$. The Bonn series showed that their surgical procedure changed from a standard temporal lobectomy to more tailored type lesionectomy over the years, and the seizure outcome improved during the same period ${ }^{31)}$. Other studies also reported no or very few advantages of extended lesionectomy over tumor resection ${ }^{12,20,52)}$. Intraoperative electrocorticography (ECoG) may be useful for detecting the epileptogenic cortex, but the impact on seizure outcome is variable ${ }^{20,49)}$. It appears that surgical outcome depends more on the pathology than on the choice of surgical procedure if the GTR of the tumor is guaranteed. Some LEATs are not easily differentiated from FCD on MRI. Because epilepsy surgery using invasive monitoring and ECoG is usually required for FCD, differential diagnosis is important. ${ }^{11} \mathrm{C}$-methionine positron emission tomography (PET) can be helpful because ganglioglioma and DNET show higher methionine uptake than $\mathrm{FCD}^{11,41)}$.

Many pediatric brain tumors have only one driver mutation. As we have seen, epilepsy-associated tumors, including many LEATs and pediatric-type DLGG, have a key driver mutation in BRAF, FGFR1, or MYB/MYBL1. BRAF is a canonical member of the RAS-RAF-MAPK pathway. FGFR1 is an upstream receptor tyrosine kinase of this pathway, and MYB and MYBL1 are also thought to be involved in the regulation of this signaling pathway (Fig. 1) ${ }^{4}$. There is evidence that the BRAF V600E mutation acts on neuronal cells of ganglioglioma to promote epileptogenesis ${ }^{28)}$. It is also interesting that the highly epileptogenic FCD is largely driven by somatic mutations of the PI3K-AKT-mTOR pathway, with which FGFR1 and MYB/MYBL1 are also involved ${ }^{4,30)}$. Many drugs targeting the RAS-RAF-MAPK pathway, especially BRAF inhibitors, are in clinical trials for the treatment of low-grade gliomas ${ }^{25}$. Although the oncological effect of BRAF inhibitors is questionable as of yet, their efficacy for seizure control in residual BRAF-mutated glioma has not been addressed and needs future evaluation.

\section{CONCLUSION}

Epilepsy-associated tumors are generally studied in the context of low-grade gliomas due to the high frequency of seizures as symptoms of the diseases. The florid emergence of new entities of glioneuronal tumors and the concept of LEATs has greatly expanded our understanding of the clinical and pathological spectrum of tumor-related epilepsy. Recent development in genome research have further widened the horizon of epilepsy-associated tumors. Low-grade gliomas can be divided into LEATs, pediatric type DLGG, and adult-type DLGG. LEATs and pediatric type DLGG are characterized by major mutations in BRAF, FGFR1, and MYB/MYBL1. Furthermore, mutation-centered classification (also expressionand methylation-based approaches) may be more accurate in the diagnosis of these tumors because the histopathological differentiation can be difficult in many LEATs and in DLGG. Targeted therapy against the oncogenic driver mutations may be applied not only for tumor control but also for seizure control.

\section{CONFLICTS OF INTEREST}

No potential conflict of interest relevant to this article was reported.

\section{INFORMED CONSENT}

This type of study does not require informed consent.

\section{- Acknowledgements}

This research was supported by grants from the National Cancer Center, Republic of Korea (NCC-1810861-1).

\section{References}

1. Banan R, Hartmann C : The new WHO 2016 classification of brain tumors-what neurosurgeons need to know. Acta Neurochir (Wien) 159 : 403-418, 2017

2. Barkovich AJ, Guerrini R, Kuzniecky RI, Jackson GD, Dobyns WB : A developmental and genetic classification for malformations of cortical 
development: update 2012. Brain 135(Pt 5) : 1348-1369, 2012

3. Baumgarten $P$, Sarlak M, Baumgarten G, Marquardt G, Seifert V, Strzelczyk $A$, et al. : Focused review on seizures caused by meningiomas. Epilepsy Behav 88 : 146-151, 2018

4. Blümcke I, Aronica E, Becker A, Capper D, Coras R, Honavar M, et al. : Low-grade epilepsy-associated neuroepithelial tumours - the 2016 WHO classification. Nat Rev Neurol 12 : 732-740, 2016

5. Blümcke I, Luyken C, Urbach H, Schramm J, Wiestler OD : An isomorphic subtype of long-term epilepsy-associated astrocytomas associated with benign prognosis. Acta Neuropathol 107 : 381-388, 2004

6. Blumcke I, Spreafico R, Haaker G, Coras R, Kobow K, Bien CG, et al. : Histopathological findings in brain tissue obtained during epilepsy surgery. N Engl J Med 377 : 1648-1656, 2017

7. Bonney PA, Boettcher LB, Conner AK, Glenn CA, Briggs RG, Santucci $J A$, et al. : Review of seizure outcomes after surgical resection of dysembryoplastic neuroepithelial tumors. J Neurooncol 126 : 1-10, 2016

8. Chappé C, Padovani L, Scavarda D, Forest F, Nanni-Metellus I, Loundou A, et al. : Dysembryoplastic neuroepithelial tumors share with pleomorphic xanthoastrocytomas and gangliogliomas BRAF(V600E) mutation and expression. Brain Pathol 23 : 574-583, 2013

9. Chassoux F, Daumas-Duport C : Dysembryoplastic neuroepithelial tumors: where are we now? Epilepsia 54 Suppl 9 : 129-134, 2013

10. Chassoux F, Rodrigo S, Mellerio C, Landré E, Miquel C, Turak B, et al. : Dysembryoplastic neuroepithelial tumors: an MRI-based scheme for epilepsy surgery. Neurology 79 : 1699-1707, 2012

11. Chong S, Phi JH, Lee JY, Kim SK : Surgical treatment of lesional mesial temporal lobe epilepsy. J Epilepsy Res 8 : 6-11, 2018

12. Clusmann H, Schramm J, Kral T, Helmstaedter C, Ostertun B, Fimmers R, et al. : Prognostic factors and outcome after different types of resection for temporal lobe epilepsy. J Neurosurg 97 : 1131-1141, 2002

13. Compton JJ, Laack NN, Eckel LJ, Schomas DA, Giannini C, Meyer FB : Long-term outcomes for low-grade intracranial ganglioglioma: 30-year experience from the Mayo Clinic. J Neurosurg 117 : 825-830, 2012

14. Daghistani R, Miller E, Kulkarni AV, Widjaja E : Atypical characteristics and behavior of dysembryoplastic neuroepithelial tumors. Neuroradiology 55 : 217-224, 2013

15. Daumas-Duport $C$ : Dysembryoplastic neuroepithelial tumours. Brain Pathol 3 : 283-295, 1993

16. Daumas-Duport C, Scheithauer BW, Chodkiewicz JP, Laws ER Jr, Vedrenne $C$ : Dysembryoplastic neuroepithelial tumor: a surgically curable tumor of young patients with intractable partial seizures. Report of thirty-nine cases. Neurosurgery $23:$ 545-556, 1988

17. Daumas-Duport C, Varlet P, Bacha S, Beuvon F, Cervera-Pierot P, Chodkiewicz JP : Dysembryoplastic neuroepithelial tumors: nonspecific histological forms -- a study of 40 cases. J Neurooncol 41 : 267-280, 1999

18. Dougherty MJ, Santi M, Brose MS, Ma C, Resnick AC, Sievert AJ, et al. : Activating mutations in BRAF characterize a spectrum of pediatric lowgrade gliomas. Neuro Oncol 12 : 621-630, 2010

19. Dudley RW, Torok MR, Gallegos DR, Mulcahy-Levy JM, Hoffman LM, Liu AK, et al. : Pediatric low-grade ganglioglioma: epidemiology, treatments, and outcome analysis on 348 children from the surveillance, epidemiology, and end results database. Neurosurgery 76 : 313-319; discussion 319; quiz 319-320, 2015

20. Englot DJ, Berger MS, Barbaro NM, Chang EF : Factors associated with seizure freedom in the surgical resection of glioneuronal tumors. Epilepsia $53:$ 51-57, 2012

21. Feindel $\mathbf{W}:$ Osler vindicated: glioma of the leg center with Jacksonian epilepsy; removal and cure, with a 50-year follow-up. Historical vignette. J Neurosurg 111 : 293-300, 2009

22. Goel NJ, Abdullah KG, Lang SS : Outcomes and prognostic factors in pediatric oligodendroglioma: a population-based study. Pediatr Neurosurg $53: 24-35,2018$

23. Harvey AS, Jayakar P, Duchowny M, Resnick T, Prats A, Altman N, et al. : Hemifacial seizures and cerebellar ganglioglioma: an epilepsy syndrome of infancy with seizures of cerebellar origin. Ann Neurol $40: 91-98$, 1996

24. Holthausen $H$, Pieper $T$, Kudernatsch $M$ : Towards early diagnosis and treatment to save children from catastrophic epilepsy -- focus on epilepsy surgery. Brain Dev 35 : 730-741, 2013

25. Kaley T, Touat M, Subbiah V, Hollebecque A, Rodon J, Lockhart AC, et al. : BRAF inhibition in BRAFV600-mutant gliomas: results from the VEBASKET study. J Clin Oncol 36 : 3477-3484, 2018

26. Kim NR, Wang KC, Bang JS, Choe G, Park Y, Kim SK, et al. : Glioblastomatous transformation of ganglioglioma: case report with reference to molecular genetic and flow cytometric analysis. Pathol Int 53 : 874882,2003

27. Koelsche C, Wöhrer A, Jeibmann A, Schittenhelm J, Schindler G, Preusser $\mathrm{M}$, et al. : Mutant BRAF V600E protein in ganglioglioma is predominantly expressed by neuronal tumor cells. Acta Neuropathol 125 : 891-900, 2013

28. Koh HY, Kim SH, Jang J, Kim H, Han S, Lim JS, et al. : BRAF somatic mutation contributes to intrinsic epileptogenicity in pediatric brain tumors. Nat Med 24 : 1662-1668, 2018

29. Kreiger PA, Okada Y, Simon S, Rorke LB, Louis DN, Golden JA : Losses of chromosomes $1 p$ and $19 q$ are rare in pediatric oligodendrogliomas. Acta Neuropathol 109 : 387-392, 2005

30. Lim JS, Kim WI, Kang HC, Kim SH, Park AH, Park EK, et al. : Brain somatic mutations in MTOR cause focal cortical dysplasia type II leading to intractable epilepsy. Nat Med 21 : 395-400, 2015

31. Luyken C, Blümcke I, Fimmers R, Urbach $H$, Elger CE, Wiestler OD, et al. : The spectrum of long-term epilepsy-associated tumors: long-term seizure and tumor outcome and neurosurgical aspects. Epilepsia 44 : 822-830, 2003

32. Maher CO, White JB, Scheithauer BW, Raffel C : Recurrence of dysembryoplastic neuroepithelial tumor following resection. Pediatr Neurosurg $44: 333-336,2008$

33. Majores $M$, von Lehe $M$, Fassunke J, Schramm J, Becker AJ, Simon M : Tumor recurrence and malignant progression of gangliogliomas. Cancer $113: 3355-3363,2008$

34. Marucci G, de Biase D, Visani M, Giulioni M, Martinoni M, Volpi L, et al. : Mutant BRAF in low-grade epilepsy-associated tumors and focal cortical dysplasia. Ann Clin Transl Neurol 1 : 130-134, 2014 
35. Ni HC, Chen SY, Chen L, Lu DH, Fu YJ, Piao YS : Angiocentric glioma: a report of nine new cases, including four with atypical histological features. Neuropathol Appl Neurobiol 41 : 333-346, 2015

36. Pallud J, Audureau E, Blonski M, Sanai N, Bauchet L, Fontaine $D$, et al. : Epileptic seizures in diffuse low-grade gliomas in adults. Brain 137(Pt 2) : 449-462, 2014

37. Pallud J, McKhann GM : Diffuse low-grade glioma-related epilepsy. Neurosurg Clin N Am 30 : 43-54, 2019

38. Pekmezci M, Villanueva-Meyer JE, Goode B, Van Ziffle J, Onodera C, Grenert JP, et al. : The genetic landscape of ganglioglioma. Acta Neuropathol Commun $6: 47,2018$

39. Perkins SM, Mitra N, Fei W, Shinohara ET : Patterns of care and outcomes of patients with pleomorphic xanthoastrocytoma: a SEER analysis. J Neurooncol 110 : 99-104, 2012

40. Phi JH, Kim SK, Cho BK, Lee SY, Park SY, Park SJ, et al. : Long-term surgical outcomes of temporal lobe epilepsy associated with low-grade brain tumors. Cancer 115 : 5771-5779, 2009

41. Phi JH, Paeng JC, Lee HS, Wang KC, Cho BK, Lee JY, et al. : Evaluation of focal cortical dysplasia and mixed neuronal and glial tumors in pediatric epilepsy patients using 18F-FDG and 11C-methionine pet. J Nucl Med 51 : 728-734, 2010

42. Prayson RA, Fong J, Najm I : Coexistent pathology in chronic epilepsy patients with neoplasms. Mod Pathol 23 : 1097-1103, 2010

43. Prayson RA, Napekoski KM : Composite ganglioglioma/dysembryoplastic neuroepithelial tumor: a clinicopathologic study of 8 cases. Hum Pathol 43 : 1113-1118, 2012

44. Qaddoumi I, Orisme W, Wen J, Santiago T, Gupta K, Dalton JD, et al. : Genetic alterations in uncommon low-grade neuroepithelial tumors: BRAF, FGFR1, and MYB mutations occur at high frequency and align with morphology. Acta Neuropathol 131 : 833-845, 2016

45. Raghavan R, Balani J, Perry A, Margraf L, Vono MB, Cai DX, et al. : Pediatric oligodendrogliomas: a study of molecular alterations on $1 p$ and $19 q$ using fluorescence in situ hybridization. J Neuropathol Exp Neurol
62 : 530-537, 2003

46. Ramkissoon LA, Horowitz PM, Craig JM, Ramkissoon SH, Rich BE, Schumacher SE, et al. : Genomic analysis of diffuse pediatric low-grade gliomas identifies recurrent oncogenic truncating rearrangements in the transcription factor MYBL1. Proc Natl Acad Sci U S A 110 : 81888193, 2013

47. Rivera B, Gayden T, Carrot-Zhang J, Nadaf J, Boshari T, Faury D, et al. : Germline and somatic FGFR1 abnormalities in dysembryoplastic neuroepithelial tumors. Acta Neuropathol 131 : 847-863, 2016

48. Rodriguez FJ, Tihan T, Lin D, McDonald W, Nigro J, Feuerstein B, et al. : Clinicopathologic features of pediatric oligodendrogliomas: a series of 50 patients. Am J Surg Pathol 38 : 1058-1070, 2014

49. Southwell DG, Garcia PA, Berger MS, Barbaro NM, Chang EF : Longterm seizure control outcomes after resection of gangliogliomas. Neurosurgery 70 : 1406-1413; discussion 1413-1414, 2012

50. Stone TJ, Keeley A, Virasami A, Harkness W, Tisdall M, Izquierdo Delgado $E$, et al. : Comprehensive molecular characterisation of epilepsyassociated glioneuronal tumours. Acta Neuropathol 135 : 115-129, 2018

51. Thom M, Blümcke I, Aronica E : Long-term epilepsy-associated tumors. Brain Pathol 22 : 350-379, 2012

52. Tomita T, Volk JM, Shen W, Pundy T : Glioneuronal tumors of cerebral hemisphere in children: correlation of surgical resection with seizure outcomes and tumor recurrences. Childs Nerv Syst 32 : 1839-1848, 2016

53. You G, Sha ZY, Yan W, Zhang W, Wang YZ, Li SW, et al. : Seizure characteristics and outcomes in 508 Chinese adult patients undergoing primary resection of low-grade gliomas: a clinicopathological study. Neuro Oncol $14: 230-241,2012$

54. Zhang J, Wu G, Miller CP, Tatevossian RG, Dalton JD, Tang B, et al. : Whole-genome sequencing identifies genetic alterations in pediatric low-grade gliomas. Nat Genet 45 : 602-612, 2013 